\title{
Platelet Transfusion Practice in Dengue Epidemic; Current Trends and Challenges - an Institutional Study
}

Soumya $\mathbf{M H}^{*}$, Das $\mathbf{S}$ and Kalyani R

Department of Pathology, Sri Devaraj Urs Medical College, Sri Devaraj Urs Academy, India

\begin{abstract}
Introduction: Dengue is an arboviral disease with an inherited risk associated with the transfusion of blood components and to prevent unnecessary transfusion during dengue epidemic a standard criteria has to be followed.

Aims/objectives: To record clinical features, laboratory investigations and management of hospitalized seropositive dengue patients. To review the appropriateness of platelet transfusion practices in order to ensure optimal utilization of platelets.

Material and methods: The Retrospective study is being done at RL Jalappa Medical Hospital from April 2015 to June 2019 on seropositive dengue cases. All serologically confirmed dengue cases who received platelet transfusion were included in the study. Patient's clinical data and platelet counts were obtained from platelet requisition forms and Medical Record Department. Case definition of Dengue/DHF/DSS applied in the present study was as recommended by WHO4 i.e. Guidelines for platelet transfusion in R. L. Jallappa Hospital Hospital were utilized as the criteria to assess the appropriateness of platelet transfusion adapted from British Committee for Standardization in Hematology (BCSH) has recommended a platelet count $<10000 / \mathrm{cmm}$ for prophylactic platelet transfusion in those with no other risk factors which would increase the risk of bleeding
\end{abstract}

Statistical analysis: Data management and analysis was done by SPSS version 22.0.

Results: During study period total of 1361 cases were diagnosed as dengue infection (out of which 757-males, 604-females). Maximum cases were seen in the age group of 11-18. All patients were categorized as per WHO dengue case classification into Dengue Fever, Dengue Haemorrhagic Fever, and Dengue Shock syndrome. In which $72.5 \%$ of patients were DF, $23.4 \%$ were DHF and $4 \%$ of DSS. In present study, maximum number of Patients and platelets transfused was seen when platelet count was $11-20 \times 1000 / \mathrm{cmm}$ that is 777 units in which RDP was 697 and SDP was 80 and lowest number of platelets transfused was seen when platelet count was $>60$. A total of 2705 RDP and 359 SDP were transfused to 1361 patients, of these 1361 patients, 316 bleeding patient received PT and 1045 non bleeding patient received prophylactic platelet transfusion in which 140 patient was requiring actual platelet transfusion and 905 patients were not needed.

Conclusion: Inappropriate usage of platelets leads to shortage of platelets. Strict adherence to British Committee for Standardization in Hematology (BCSH) Guidelines will optimize platelet usage with this true DHF and DSS will be benefited during epidemic outbreaks. Educating patients and Patients attenders will help reducing anxiety and this in turn help clinician for better judgment on evidence based transfusion reducing inappropriate transfusion. This study emphasis on platelet usage trends in regional areas and highlighting.

Keywords: Dengue fever; Dengue hemorrhagic fever; Dengue shock syndrome; Random donor platelet; Single donor platelet

\section{Introduction}

Dengue is an Arboviral disease caused by the dengue virus which is carried by and introduced into human host by the female Aedes mosquito [1]. The global incidence of dengue has significantly increased over the past decade and is now endemic in many developing countries [1]. WHO classifies and defined the disease as follows: Dengue Fever (DF), Dengue Hemorrhagic Fever (DHF), and Dengue Shock Syndrome (DSS). The classical DF is defined by the presence of acute febrile illness and $\geq 2$ of the following symptoms: headache, retro-orbital pain, myalgia, arthralgia and rash [2]. DHF is defined as fever with thrombocytopenia, any hemorrhagic manifestation e.g., positive tourniquet test, petechiae; purpura ecchymosis; gum bleeding; or vaginal bleeding and evidence with plasma leakage ( $\geq 20 \%$ hematocrit or by the presence of pleural or abdominal effusion or hypoalbuminemia) [2]. DSS was defined by rapid and weak pulse with narrow pulse pressure [2]. There is large lack of evidence-based platelet transfusion guidelines when patients develop bleeding symptoms in Dengue infection.
All these cause wastage of platelet component by unnecessary transfusion which can result in scarcity of platelets during dengue outbreaks and actual patients are not benefitted. In India, blood transfusion services are highly fragmented, and only $35 \%$ blood units are separated into components [3].

In dengue infection prominent feature is thrombocytopenia. The major cause of thrombocytopenia is immunological destruction of platelets which contributes to platelet dysfunction and disturbed hemostasis in dengue patients [4]. The present study was undertaken

*Corresponding author: Soumya $\mathrm{MH}$, Department of Pathology, Sri Devaraj Urs Medical College, Sri Devaraj Urs Academy, India, Tel: 8383869289; E-mail: soumyamhadimani@gmail.com

Received November 05, 2019; Accepted November 18, 2019; Published November 22, 2019

Citation: Soumya MH, Das S, Kalyani R (2019) Platelet Transfusion Practice in Dengue Epidemic; Current Trends and Challenges - an Institutional Study. J Blood Lymph 9: 255.

Copyright: (c) 2019 Soumya MH, et al. This is an open-access article distributed under the terms of the Creative Commons Attribution License, which permits unrestricted use, distribution, and reproduction in any medium, provided the original author and source are credited. 
Citation: Soumya MH, Das S, Kalyani R (2019) Platelet Transfusion Practice in Dengue Epidemic; Current Trends and Challenges - an Institutional Study. J Blood Lymph 9: 255.

Page 2 of 3

to assess and analyse the utilisation of platelet products in a tertiary care hospital during the period of April 2015 to June 2019.

\section{Methodology}

Case definition of DF/DHF/DSS applied in the present study was as recommended by WHO Guidelines for platelet transfusion in R. L. Jallappa Hospital were utilized as the criteria to assess the appropriateness of platelet transfusion adapted from British Committee for Standardization in Hematology (BCSH) which has recommended a platelet count $<10000 / \mathrm{cmm}$ for prophylactic platelet transfusion in those with no other risk factors which would increase the risk of bleeding. Grades of bleeding were evaluated as per criterion listed in Table 1 [5].

\section{Results}

During the study period, a total of 1361 patients were diagnosed of dengue infection, (757 males, 604 females) was admitted and given platelet transfusion. Maximum age group having dengue infection was seen in the age group of 10-30 years, out of which maximum cases were seen in the age group of 11-18 which may be attributed to increased exposure to environment and less knowledge about the safety measures (Figure 1).

All patients were categorized as per WHO dengue case classification into Dengue Fever, Dengue Haemorrhagic Fever and Dengue Shock Syndrome. In our study Dengue fever cases was $72.5 \%$, Dengue Haemorrhagic Fever was 23.4\%, Dengue Shock Syndrome was 4.0\% (Table 2).

\begin{tabular}{|l|l|}
\hline Category & Definition \\
\hline Minor & $\begin{array}{l}\text { Petechiae, purpura, ecchymosis, oropharyngeal/gum bleeding, } \\
\text { epistaxis, vaginal spotting, retinal haemorrhage without visual } \\
\text { impairment and any bleeding not requiring red cell transfusion, }\end{array}$ \\
\hline Major & $\begin{array}{l}\text { Melaena, haematemesis, haemoptysis, haematuria, haematochezia, } \\
\text { abnormal vaginal bleeding, musculoskeletal/soft tissue bleed,CNS } \\
\text { bleeding including retinal bleeding with visual impairment, invasive } \\
\text { site bleeding or any bleeding requiring red cell transfusion and any } \\
\text { debilitating/ fatal bleed }\end{array}$ \\
\hline
\end{tabular}

Table 1: Grades of bleeding

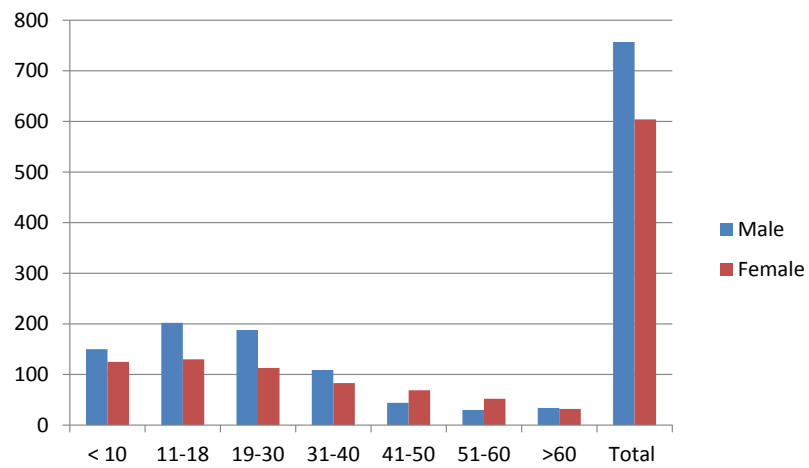

Figure 1: Age and sex distribution of dengue-affected patients.

\begin{tabular}{|l|c|c|}
\hline Category & Number & $\%$ \\
\hline Dengue Fever & 987 & 72.5 \\
\hline Dengue Haemorrhagic Fever & 319 & 23.4 \\
\hline Dengue Shock syndrome & 55 & 4.0 \\
\hline Total & 1361 & 100 \\
\hline
\end{tabular}

Table 2: Categories of dengue infection.
The diagnosis was established on clinical suspicion and confirmed by laboratory tests like ELISA and dot blot assays of non-structural protein 1 (NS1) or by commercially available immunochromatographic kits for anti-dengue IgM and IgG antibodies. In our study total no of NS1 positive cases were 652(45.90\%), IgM was 310 (22.77\%) and NS1 and Igm was 399 (29.31\%) (Table 3).

In Table 4 we have divided total no of patients having different platelet counts ranging from $<10$ to $>60 \times 1000 / \mathrm{cmm}$ and observed how many units of platelets transfused, out of which how many patients were transfused with RDP (Random Donor Platelet) and SDP (Single Donor Platelet). In present study, maximum number of Patients transfused with platelets was seen when platelet count was 11$20 \times 1000 / \mathrm{cmm}$ that is 777 units in which RDP was 697 and SDP was 80 . Lowest number of platelets transfused was seen when platelet count was $>60$.

In Table 5 we have divided patients receiving platelet transfusion into bleeding and non bleeding. In Table 6 the patients are classified as bleeding and non-bleeding patients versus $\mathrm{BCSH}$ guidelines followed and unfollowed. Total no of bleeding patients was 316 and nonbleeding was 1045 . We observed that maximum no of bleeding patients was seen with platelet count of $11-20 \times 1000 / \mathrm{cmm}$ and maximum

\begin{tabular}{|l|c|c|}
\hline & Total no of cases & $\%$ \\
\hline NS1 Positive & 652 & 45.90 \\
\hline IgM Positive & 310 & 22.77 \\
\hline NS1 and IgM Positive & 399 & 29.31 \\
\hline Total & 1361 & 100 \\
\hline
\end{tabular}

Table 3: Patients with NS1 Positive, IgM positive, both NS1 and IgM positive and negative cases.

\begin{tabular}{|c|c|c|c|c|}
\hline $\begin{array}{c}\text { Platelet counts } \\
\times\end{array}$ & No of patients & $\begin{array}{c}\text { No of units } \\
\text { of platelets } \\
\text { transfused }\end{array}$ & RDP & SDP \\
\hline$<10$ & 230 & 664 & 566 & 98 \\
\hline $11-20$ & 302 & 777 & 697 & 80 \\
\hline $21-30$ & 326 & 434 & 399 & 35 \\
\hline $31-40$ & 152 & 364 & 296 & 68 \\
\hline $41-50$ & 160 & 359 & 315 & 44 \\
\hline $51-60$ & 90 & 232 & 200 & 32 \\
\hline$>60$ & 101 & 234 & 232 & 2 \\
\hline Total & 1361 & 3064 & 2705 & 359 \\
\hline
\end{tabular}

Table 4: Platelet counts and platelet transfusion details.

\begin{tabular}{|c|c|c|c|}
\hline Platelet count 10$/ \mathbf{I}$ & \multicolumn{3}{|c|}{ Number of patients } \\
\hline & Total & Bleeding & Non-bleeding \\
\hline$<10$ & 230 & 90 & 140 \\
\hline $11-20$ & 302 & 110 & 192 \\
\hline $21-30$ & 326 & 50 & 276 \\
\hline $31-40$ & 152 & 23 & 129 \\
\hline $51-50$ & 160 & 29 & 131 \\
\hline$>60$ & 90 & 11 & 79 \\
\hline Total & 101 & 3 & 98 \\
\hline
\end{tabular}

Table 5: Platelet counts of patients receiving platelet transfusion (Bleeding and Non-Bleeding).

\begin{tabular}{|l|c|c|}
\hline & Bleeding & Non bleeding \\
\hline Guidelines fulfilled & 316 & 140 \\
\hline Guidelines unfulfilled & - & 905 \\
\hline
\end{tabular}

Table 6: Number of patients following BCSH Guidelines. 
Citation: Soumya MH, Das S, Kalyani R (2019) Platelet Transfusion Practice in Dengue Epidemic; Current Trends and Challenges - an Institutional Study. J Blood Lymph 9: 255.

Page 3 of 3

\begin{tabular}{|c|c|c|c|}
\hline \multirow{2}{*}{ Blood group } & \multicolumn{3}{|c|}{ Patients } \\
\cline { 2 - 4 } & \%DF (72.5) & \% DHF (23.4) & \%DSS (4) \\
\hline A & 218 & 153 & 10 \\
\hline B & 101 & 222 & 13 \\
\hline AB & 56 & 51 & 7 \\
\hline
\end{tabular}

Table 7: Association between different blood groups and forms of dengue.

no of non-bleeding cases was seen with platelet count 21-30 × 1000/ $\mathrm{cmm}$. Patients who fulfilled British Committee for Standardization in Hematology (BCSH) Guidelines was 316 bleeding patients, out of 1045 non-bleeding patients, 140 patients with platelet count $<10,000 / \mathrm{cmm}$ followed guidelines and prophylactic platelet transfusion was done. 905 non-bleeding patients received platelet transfusion unnecessarily and did not follow guidelines leading to wastage of platelet component (Table 7).

In present study we divided DF, DHF and DSS into different blood groups and observed association between different blood groups and forms of dengue. Maximum no of patient who had DF was of A blood group, severe forms of dengue like DHF and DSS was associated with O blood group.

\section{Discussion}

In present study total 1361 cases with Dengue infection was transfused platelets ( 757 males, 604 females). Male predominance was seen similar to that reported in other studies [5-8]. In present study maximum number of patients was in the age group of 11-18years i.e. pediatric age group predominance was seen, which may be attributed to increased exposure to environment and less knowledge about the safety measures, while study done by Yashaswini et al. [9] shows adult predominance.

In present study dengue infections included DF (72.5\%), DHF (23.4\%), DSS (4.0). In a study by Rahul et al 531 dengue positive cases were given platelet transfusion of which 457(86.0\%) DF, 19 (6.4\%) DHF and 21 (4\%) DSS. Present study has more number of DHF and less number of DF. The difference may be attributed to better reporting of bleeding status in present study.

In present study 2705 RDPs and 359 SDPs were transfused to 1361 patients. Rahul et al. reported transfusion of 1750 RDPs and 114 SDPs unit of platelets transfused to 531 patients. The higher amounts of platelet transfusion in present study may be because of larger number of bleeding patient (200) with platelet count $<20 \times 10^{9} / 1$ in present study who were appropriate candidates for platelet transfusion according to BCSH. Non-bleeding 905 patient was received inappropriate platelet transfusion. Causes of bleeding other than dengue were ruled out before initiation of platelet transfusion in all patients [10]. In present study maximum number of patients with O blood group has DHF and DSS. In contrast to present study, study done by Kalamathy et al. showed high number of cases with $\mathrm{AB}$ blood group having severe forms of Dengue like DHF and DSS.

\section{Conclusion}

Inappropriate usage platelets for stable patients with low platelet count causes decreased availability of platelets, Consequently, with best efforts by blood bank the sever forms of Dengue patients with DHF/DSS might not get intended dose of platelets. There is a need for decreasing unwanted transfusions, which can be achieved by strict adherence to British Committee for Standardization in Hematology (BCSH) Guidelines. Educating patients and Patients attenders will help in reducing anxiety and this in turn help clinician for better judgement on evidence based transfusion reducing inappropriate transfusion. This study highlight the trends of platelet usage in Kolar and adjoining areas, with emphasis on appropriate and inappropriate usage, which in turn may help in identifying the deficiencies in platelet transfusion services and also help in formulating Indian Guidelines to render better patient care based on regional scenarios.

\section{References}

1. Sinha N, Gupta N, Jhamb R, Gulati S, Ajit VK (2008) The 2006 dengue outbreak in Delhi, India. J Commun Dis 40: 243-248.

2. World Health Organization (2009) Dengue: guidelines for diagnosis, treatment prevention and control.

3. Choudhury N (2011) Blood transfusion in borderless South Asia. Asian J Transfus Sci 5: 117-120.

4. Mitrakul C, Poshyachinda M, Futrakul P, Sangkawibha N, Ahandrik S (1977) Hemostatic and platelet kinetic studiesin dengue hemorrhagic fever. Am J Trop Med Hyg 26: 975-984.

5. Chaurasia R, Zaman S ,Chatterjee K, Das B (2015) Retrospective Review of Platelet Transfusion Practices during 2013 Dengue Epidemic of Delhi, India. Transfus Med Hemother 42: 227-231.

6. Hammond SN, Balmaseda A, Perez L, Tellez Y, Saborio SI, et al. (2005) Differences in dengue severity in infants, children,and adults in a 3-year hospital-based study in Nicaragua. Am J Trop Med Hyg 73: 1063-1070.

7. Bandyopadhyay B, Bhattacharyya I, Adhikary S, KonarJ, Dawar N, et al. (2013) A comprehensive study on the 2012 dengue fever outbreak in Kolkata, India. ISRN Virol 2013: 5.

8. Saqib MA, Rafique I, Bashir S, Salam AA (2014) A retrospective analysis of dengue fever case management and frequency of co-morbidities associated with deaths. BMC Res Notes 7: 205.

9. Yashaswini LS, Priya (2017) A study of hematological parameters and requirement of platelet transfusion in dengue fever. Int J Adv Med 4: 16682167.

10. Murugananthan K, Subramaniyam S, Kumanan T, Owens L, Ketheesan N, et al. (2018) Blood group $A B$ is associated with severe forms of dengue virus infection. Virus Disease 29: 103-105. 